Selim Orgül

George A. Croffi

E. Michael Van Buskirk

\section{Variability of contour line alignment on sequential images with the Heidelberg Retina Tomograph}

Received: 14 June 1996

Revised version received: 1 October 1996 Accepted: 14 October 1996
S. Orgül · G.A. Croffi

E.M. Van Buskirk

Devers Eye Institute,

R.S. Dow Neurological Science Institute, Legacy Portland Hospitals, Portland, Oregon, USA

\section{S. Orguil (}

University Eye Clinic Basel, Mittlere Straße 91, P.O. Box, CH-4012 Basel, Switzerland Tel. +41-61-3217777; fax: +41-61-3217763; e-mail: ORGUEL@ubaclu.unibas.ch
Abstract - Background: The influence of the contour line alignment software algorithm on the variability of the Heidelberg Retina Tomograph (HRT) parameters remains unclear. Methods: Nine discrete topographic images were acquired with the HRT from the right eye in six healthy, emmetropic subjects. The variability of topometric data obtained from the same topographic image, analyzed within different samples of images, was evaluated. A total of four mean topographic images was computed for each subject from: all nine discrete images (A), the first six of those images (B), the last six of those nine images $(C)$, and the first three combined with the last three images (D). A contour line was computed on the mean topographic image generated from the nine discrete topographic images (A). This contour line was then applied to the three other mean topographic images (B, $\mathrm{C}$, and $\mathrm{D}$ ), using the contour line alignment in the HRT software. Subsequently, the contour line on the mean topographic images was applied to each of the discrete members of the particular images subsets used to compute the mean topographic image, and the topometric data for these discrete topographic images was computed successively for each subset. Prior to processing each subset, the contour line on the discrete topo- graphic images was deleted. This strategy provided a total of three analyses on each discrete topographic image: as a member of the nine images (mean topographic image $\mathrm{A}$ ), and as a member of two subsets of images (mean topographic image B, C, and/or D). The coefficient of variation $(100 \times \mathrm{SD} /$ mean) of the topographic parameters within those three analyses was calculated for each discrete topographic image in each subject " intraimage" coefficient of variation). In addition, a coefficient of variation between the nine discrete topographic images ("interimage" coefficient of variation) was calculated. - Results: The "intraimage" and "interimage" variability for the various topographic parameters ranged between $0.03 \%$ and $3.10 \%$ and between $0.03 \%$ and $24.07 \%$ respectively. The "intraimage" coefficients of variation and "interimage" coefficients of variation correlated significant $\left(r^{2}=0.77 ; P<0.0001\right)$. - Conclusion: A high "intraimage" variability, i.e. a high variability in contour line alignment between sequential images, might be an important source of test re-test variability between sequential images. 


\section{Introduction}

Glaucomatous morphological changes of the optic nerve may precede measurable visual field defects by several years [10]. Therefore, reliable evaluation of the optic nerve head topography could prove important in the assessment and the management of glaucoma patients, especially in early stages of the disease. Scanning-laser ophthalmoscopy is a recently developed method to image and quantify the topographic structure of the retina and the optic nerve $[1-9,11]$. The Heidelberg Retina Tomograph (HRT) is a scanning-laser ophthalmoscope that utilizes confocal optics to produce three-dimensional topographic images. Topometric data can be computed on these images.

The test re-test behavior of the scanning-laser ophthalmoscope has been evaluated thoroughly $[1,2,4-9$, 11]. The influence of the contour line alignment software algorithm on the variability of the HRT parameters, however, remains unclear. The HRT stores a defined contour line for repeated analysis of the images or alignment on sequential images, allowing those images to be analyzed with respect to the same region of interest. The software of the HRT allows generation of a mean topographic image from a set of discrete topographic images by determining the average height measurement at each image location (pixel). Once a region of interest has been defined by a contour line on a mean topographic image, this contour line can be transferred ("imported") onto the discrete topographic images included to compute the mean topographic image. Consequently, topometric data can be computed for each discrete topographic image, and the mean values of topometric data in sequential samples of topographic images can be compared statistically. Ideally, during this process, the alignment of the contour line on discrete topographic images would be independent of the other discrete topographic images included to compute the mean topographic image. The purpose of this study was to determine the variability of the alignment of the contour line, i.e. the variability of topometric data obtained from the same topographic image, analyzed within different samples of images.

\section{Subjects and methods}

The variability of topometric data obtained from the same topographic image, analyzed within different samples of images, was evaluated among six healthy, 28- to 41 year-old human subjects (four women and two men). The configuration of the optic disc was relatively uniform among the six volunteers with mainly small temporal flat cups. The Heidelberg software, version 1.11 (Heidelberg Engineering, Heidelberg, Germany) was used for the present
Table 1 Topographic parameters provided by the Heidelberg Retina Tomograph
Area

Effective area

Area below reference

Mean radius

Mean height of contour

Height variation contour

Volume below reference

Volume above reference

Volume below surface

Volume above surface

Mean depth in contour

Effective mean depth

Maximum depth in contour

Third moment in contour
Total area within the contour line

Total area of those parts within the contour line which are located below the curved surface

Total area of those parts within the contour line which are located below the reference plane

Mean radius of the contour line

Mean coordinate of the corrected (for crossing vessels) contour line on the axis toward the vitreous

Difference in height between the most elevated and the most depressed point of the corrected contour line

Total volume of those parts within the contour line which are located below the reference plane

Total volume of those parts within the contour line which are located above the reference plane

Total volume of those parts within the contour line which are located below the curved surface

Total volume of those parts within the contour line which are located above the curved surface

Mean depth relative to the curved surface of the whole structure within the contour line (parts located above the curved surface contribute with a negative value)

Mean depth relative to the curved surface of all parts within the contour line which are located below the curved surface

Mean depth relative to the curved surface of the $5 \%$ picture elements with the highest depth values within the contour line

Parameter describing the overall shape of the optic nerve head 


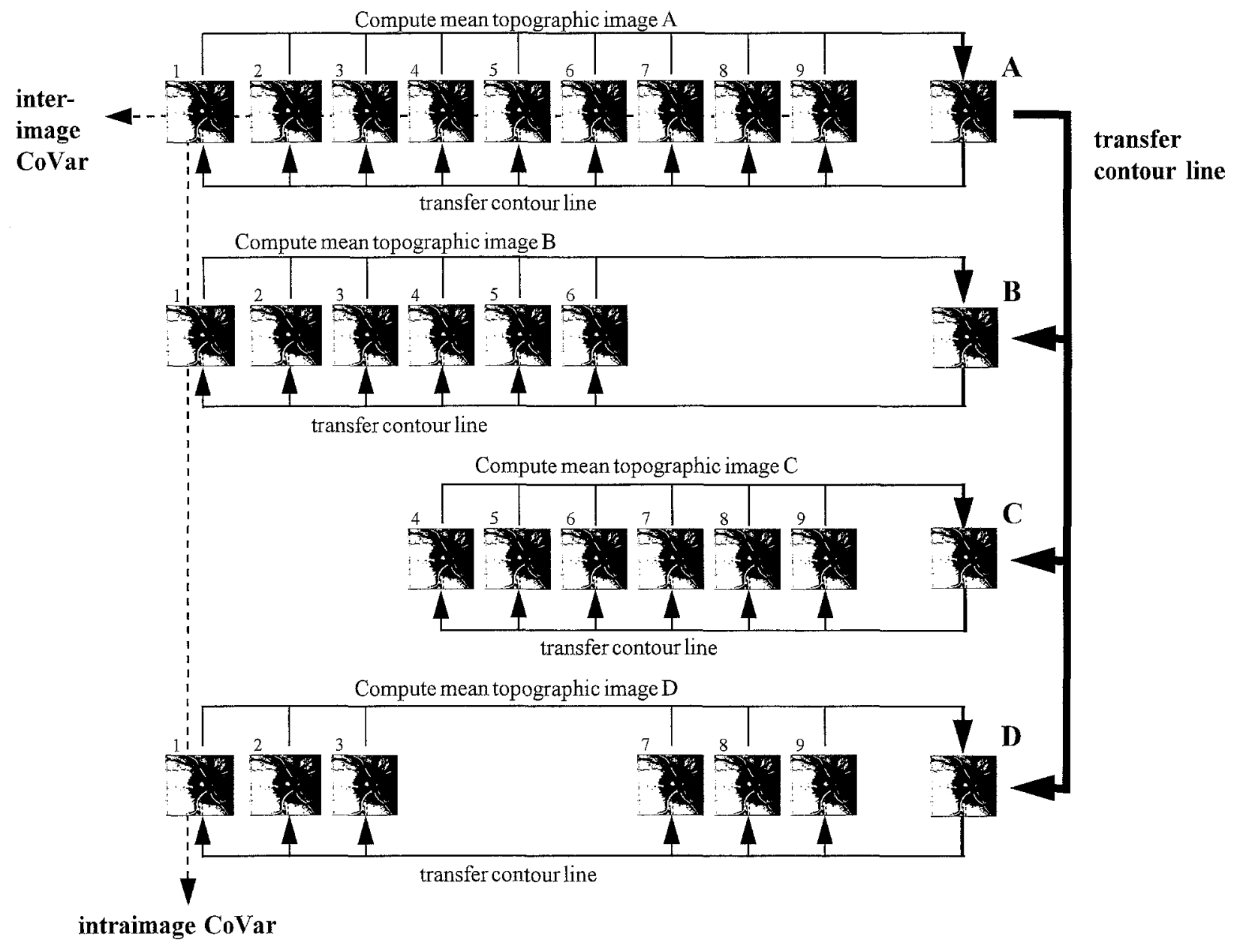

Fig. 1 Description of the experimental procedure in one subject. Nine discrete topographic images were acquired. A total of four mean topographic images were computed from: $A$ all nine discrete images; $B$ the first six of those nine images; $C$, the last six of those nine images; and $D$ the first three combined with the last three images. A contour line was computed on the mean topographic image generated from the nine discrete topographic images (A). This contour line was then applied to the three other mean topographic images $(\mathrm{B}-\mathrm{D})$, using the contour line alignment algorithm in HRT software. Subsequently, the contour line on the mean topographic images was applied to each of the discrete members of the particular image subsets used to compute the mean topographic image, and the topometric data for these discrete topographic images were computed successively for each subset. Prior to processing each subset, the contour line on the discrete topographic images was deleted. Consequently, for each one of the nine discrete topographic images, a total of three analyses were obtained: as a member of the nine images (mean topographic image $\mathbf{A}$ ), and as a member of two subsets of images (mean topographic image B, C, and/or D). The coefficient of variation (CoVar $=100 \times \mathrm{SD} / \mathrm{mean})$ of the topographic parameters within those three analyses was calculated for each discrete topographic image ("intraimage" coefficient of variation). These values were averaged to obtain an average "intraimage" coefficients of variation (average "intraimage" CoVar) for each subject and for each topographic parameter. In addition, an "interimage" coefficient of variation ("interimage" CoVar) between the nine discrete topographic images of the data set A was calculated analysis. After informed consent had been obtained, nine discrete topographic images, numbered sequentially from 1 to 9 , were acquired with the HRT from the right eye of six healthy, emmetropic subjects in direct sequence, the subjects leaning back in their chair between images. During the imaging, the subjects fixated on a distant target with the left eye. The same scan focus and scan depth was used for all sequential images in a given subject [4]. A $10 \times 10^{\circ}$ field size was used for the recordings, and all images were obtained through an undilated pupil.

For each one of the nine discrete images recorded in each subject, a total of three analyses were obtained. To this end, a total of four mean topographic images were computed for each subject: all nine discrete images (mean topographic image A, images 1-9), the first six of those nine images (mean topographic image $B$, images $1-6$ ), the last six of those nine images (mean topographic image $C$, images 4-9), and the first three combined with the last three images (mean topographic image D, images 1-3, 7-9). Each discrete image was part of the mean topographic image containing all nine images and of two additional subgroups containing six images. The experimental procedure is illustrated in Fig. 1. On the mean topographic image including all nine images (A), the region of interest was defined by a contour line to best approximate the margin of the optic disc (inner edge of Elschnig's scleral ring) [9]. This contour line was then applied to the three other mean topographic images (B, C, and D), using the contour line alignment algorithm in the HRT software. Subsequently, the contour line on the mean topographic images was applied to each of the discrete members of the particular image subsets used to compute the mean topographic image, and the topometric data for these dis- 
crete topographic images were computed successively for each subset. Prior to processing each subset, the contour line on the discrete topographic images was detected. The same procedure was applied to the nine discrete topographic images for each of the six subjects.

All stereometric measurements with the HRT are made within a region of interest which is enclosed by the contour line. For the evaluation of volumetric parameters, the upper limitation towards the vitreous is provided either by a curved surface which follows the height variation along the contour line [9], or by a planar surface referred to as the reference plane. For parameters defined by the planar reference plane, earlier versions of the HRT software used a plane $320 \mu \mathrm{m}$ below the mean retinal height. The software version 1.11 provides a reference plane $50 \mu \mathrm{m}$ below the mean height of the contour line beween -4 and $-10 \mathrm{deg}$. For the current investigation, this new reference plane was used. The parameters provided by the HRT are described in Table 1.

The design described above provided a total of three analyses on each discrete topographic image: as a member of the nine images (the mean topographic image $\mathrm{A}$ ), and as a member of two subsets of images (the mean toporaphic image $B, C$, and/or D). The coefficient of variation $(100 \times \mathrm{SD} /$ mean) among the three analyses on one given discrete topographic image ("intraimage" coefficient of variation) was calculated for each topographic parameter. "Intraimage" coefficients of variation were obtained in all nine images for each topographic parameter for each subject. Average values of these nine "intraimage" coefficients of variation were determined for each subject and for each topographic parameter. Finally, the "intraimage" coefficients of variation obtained in all six subjects were average to obtain a mean "intraimage" coefficient of variation for each topographic parameter. An "interimage" coefficient of variation was calculated with the data from the set with all nine discrete topographic images for each subjects and for each topographic parameter. The "interimage" coefficients of variation obtained in all six subjects were averaged to obtain a mean "interimage" coefficient of variation for each topographic parameter.

\section{Results}

Among the mean topographic images A (each mean topographic image $\mathrm{A}$ was computed with all nine discrete topographic images acquired on one subject), the mean $( \pm \mathrm{SD})$ standard deviation of the height measurements at a pixel was $31.21 \pm 4.13 \mu \mathrm{m}$. The mean "intraimage" coefficients of variation and mean "interimage" coefficients of variation obtained for the six subjects are listed in Table 2 for all the topographic parameters. The mean "intraimage" coefficient of variation values ranged between $0.03 \%$ and $3.10 \%$. The mean "interimage" coefficient of variation values ranged between $0.03 \%$ and $24.07 \%$. A linear correlation between "intraimage" coefficients of variation and "interimage" coefficients of variation was statistically highly significant (Pearson's linear correlation factor $r^{2}=0.77 ; P<0.0001$ ) with a slope of the correlation line of 0.11 . A residual analysis showed no outliers and the residuals were normally distributed (Shapiro Wilk's $W=0.93 ; P=0.27$ ). This linear correlations suggests that the interimage variability is approximately ten times higher than the intraimage variability.
Table 2 Average coefficients of variation (100×SD/mean) of topometric data within images and between images obtained in normal, emmetrope eyes with the Heidelberg Retina Tomograph

\begin{tabular}{llc}
\hline $\begin{array}{l}\text { Topographic } \\
\text { parameter }\end{array}$ & $\begin{array}{l}\text { Intraimage } \\
\text { coefficient } \\
\text { of variation }\end{array}$ & $\begin{array}{c}\text { Interimage } \\
\text { coefficient } \\
\text { of variation }\end{array}$ \\
\hline Area & 0.05 & 0.05 \\
Effective area & 0.99 & 7.37 \\
Area below reference & 2.17 & 14.82 \\
Mean radius & 0.03 & 0.03 \\
Mean height of contour & 1.93 & 19.82 \\
Height variation contour & 1.81 & 17.53 \\
Volume below reference & 3.10 & 18.14 \\
Volume above reference & 2.35 & 21.72 \\
Volume below surface & 0.69 & 9.66 \\
Volume above surface & 2.21 & 24.07 \\
Mean depth in contour & 0.70 & 9.56 \\
Effective mean depth & 0.58 & 8.00 \\
Maximum depth in contour & 0.36 & 9.27 \\
Third moment in contour & 1.36 & 9.10 \\
\hline
\end{tabular}

\section{Discussion}

In the present study, the variability of topometric data obtained with the HRT from the same topographic image, analyzed within different samples of images, has been investigated. The present data indicate that topographic parameters of discrete topographic images are altered by the topographic images included in the analzyed sample, although this alteration seems to be minor compared to the overall variability.

The sources of variability with the HRT are not clear. The cardiac cycle has recently been shown to represent an important source of variability for topographic measurements with the HRT [1]. A further important factor which influences the variability of topometric data obtained in a sample of topographic images is the misalignment between the laser scanner and the patient [8]. The reproducibility of height measurements (standard deviation) at a pixel has been reported as $30.1 \mu \mathrm{m}$ in normal eyes with the HRT [6]. This image acquisition variability plays an important role in subsequent topographic measurement variability. Topographic parameters of the optic nerve and peripapillary region obtained from sequential images show coefficients of variation ranging between $4.1 \%$ and $30.6 \%$ in healthy human eyes $[7,9]$. The values of "interimage" coefficients of variation observed in the present study compare well with the range of coefficients of variation of topographic parameters reported in the literature. The present data show that image acquisition variability might be of greater importance for the computation of topometric data than the consequences of the variability of the alignment of the contour line. The values in Table 2 suggest that the image acquisition variability is approximately ten times higher than the variability of topometric data obtained from the same 
topographic image, analyzed within different samples of images.

If a contour line, drawn on a mean topographic image, is transferred onto the discrete topographic images, the topometric data computed on those images are obviously affected by the discrete topograhic images included to compute the mean topographic image. The observed variability of topometric data on a given topographic image is most probably a consequence of the variability of the alignment of the contour line. Such a variability depends on the images included in the analyzed sample, and might be due to two factors. One factor might be that slight shifts are necessary to align a contour line on a subsequent image. The coordinates of the contour line on the new image can not be fractions of a pixel. Therefore, an additional shift is necessary for the alignment of the contour line to obtain whole numbers of pixels. Such adjustments induce a slight error during the transfer of the contour line on a new image. Because the original contour line was transferred to another mean topographic image, prior to alignment on the discrete topographic images, this effect might have been amplified. Another factor might be that each discrete topograhic image influences the height at each image location in a mean topographic image. Therefore, the height along the contour of the optic nerve will slightly vary with the different discrete topographic images included to compute the mean image. Consequently, the coordinates of the original contour line will be affected by the transfer on a mean topographic image computed from a subset of discrete topographic images. During the subsequent transfer onto the discrete topographic images, a slightly different contour line will be created for each analyzed subset.
The "intraimage" coefficients of variation and the "interimage" coefficients of variation correlate linearly. Possibly, a high test-retest variability of topographic measurements potentiates the factors responsible for "intraimage" variability. In addition, a high "intraimage" variability, i.e. a high variability in contour line alignment between sequential images, might cause a high testretest variability between sequential images. Furthermore, it is striking that the "intraimage" coefficients of variation for the three parameters defined by the planar reference plane range among the four highest highest values. It can be hypothesized that the setting of the reference plane, as defined for the HRT software version 1.11 , is very sensitive to small shifts during contour line alignment between images. Such an effect might also influence the "interimage" variability. Indeed, the "interimage" variability of Area below reference and Volume below reference are notably higher than that for corresponding parameters computed by means of the curved surface. The reason why the variability of Volume above surface is much higher than that of other parameters defined by the curved surface might be that this parameter represents the smallest topometric value. Indeed, coefficients of variation for small means, in comparison to the resolution of the measurement technique, tend to be higher.

The present data suggest that a high "intraimage" variability, i.e. a high variability in contour line alignment between sequential images, might be an important source of test-retest variability between sequential images.

Acknowledgements This study was supported in part by the Schweizerische Stiftung für medizinisch-biologische Stipendien and by National Institutes of Health grant EYO5231.

\section{References}

1. Chauhan BC, McCormick TA (1995) Effect of the cardiac cycle on topographic measurements using confocal scanning laser tomography. Graefe's Arch Clin Exp Ophthalmol 233:568 572

2. Chauhan BC, LeBlanc RP, McCormick TA, Rogers JB (1994) Testretest variability of topographic measurements with confocal scanning laser tomography in patients with glaucoma and control subjects. Am J Ophthalmol 118:9-15

3. Dreher AW, Weinreb RN (1991) Accuracy of topographic measurements in a model eye with the laser tomographic scanner. Invest Ophthalmol Vis Sci 32: 2992-2996
4. Hosking SL, Flanagan JG (1996) Prospective study design for the Heidelberg Retina Tomograph: the effect of change in focus setting. Graefe's Arch Clin Exp Ophthalmol 234: 306-310

5. Kruse FE, Burk RO, Volcker HE, Zinser G, Harbarth U (1989) Reproducibility of topographic measurements of the optic nerve head with laser tomographic scanning. Ophthalmology 96: $1320-1324$

6. Lusky M, Bosem ME, Weinreb RN (1993) Reproducibility of optic nerve head topography measurements in eyes with undilated pupils. J Glaucoma 2: 104-109

7. Mikelberg FS, Wijsman K, Schulzer M (1993) Reproducibility of topographic parameters obtained with the Heidelberg Retina Tomograph. J Glaucoma 2: 101-103
8. Orgül S, Cioffi GA, Bacon DR, Van Buskirk EM (1996) Sources of variability of topometric data with a scanning laser ophthalmoscope. Arch Ophthalmol 114: 161-164

9. Rohrschneider K, Burk ROW, Völker HE (1993) Reproducibility of topometric data acquisition in normal and glaucomatous optic nerve heads with the laser tomographic scanner. Graefe's Arch Clin Exp Ophthalmol 23: 457-564

10. Sommer A, Pollack I, Maumenee AE (1979) Optic disc parameters and onset of glaucomatous field loss. 1 . Methods and progressive changes in disc morphology. Arch Ophthalmol 97: 1444-1448

11. Weinreb RN, Lusky M, Bartsch DU, Morsman D (1993) Effect of repetitive imaging on topographic measurement of the optic nerve head. Arch Ophthalmol 111:636-638 